\title{
PTH-040 PILLCAM COLON CAPSULE IS AN EFFECTIVE TOOL IN MANAGEMENT OF PATIENTS WITH SUSPECTED OR KNOWN INFLAMMATORY BOWEL DISEASE: PRELIMINARY RESULTS FROM A UK TERTIARY CARE CENTRE
}

doi:10.1136/gut.2011.239301.441

D Majumdar, ${ }^{1, *}$ R Sidhu, ${ }^{1}$ A J Lobo, ${ }^{1}$ M E McAlindon' ${ }^{1}$ Gastroenterology, Sheffield Teaching Hospitals NHS Foundation Trust, Sheffield, UK

Introduction PillCam Colon capsule endoscopy (CCE) is a relatively new technique for imaging the colon. Its role in patients with inflammatory bowel disease (IBD) has not been fully explored. A recent European multicentre trial has shown that PillCam (Given Imaging) reactivated in the small bowel $(\mathrm{SB})$ in about $98 \%$ of patients. Ileocolonic mucosal visualisation thus achieved by CCE may be a potential strategy in assessment of patients with known or suspected IBD.

Methods Consecutive patients with proven or suspected IBD were included in the study. The CCE was performed as per standard protocol (with PEG and sodium phosphate as laxative agents). Patient demographics, clinical presentation and indication for CCE as well as nature and location of the findings, colonic transit time, complications and clinical implications of the findings, were analysed. Bowel cleanliness was described using a 4 point validated scale ( 1 - excellent, 2 - good, 3 - fair, 4 - poor). Data was expressed as median and IOR.

Results 29 patients $(86.2 \%$ females) with proven $(n=10)$ or suspected IBD ( $n=19)$ underwent CCE between October 2007 and $2010.41 .4 \%$ of all patients had refused colonoscopy; $31 \%$ had a failed attempt while the rest were offered CCE as the initial imaging test. Median age was 44 (IOR 29-55.5) years. PillCam colon 1 and 2 were respectively used in $26(89.7 \%)$ and $3(11.3 \%)$ patients. CCE failed in $2(6.9 \%)$ patients, all in the known IBD patients. Complete colonic mucosal visualisation was noted in $62.1 \%$ of all patients. 8 of patients with IBD had Crohn's disease (CD) ( $\mathrm{n}=4$ Crohn's colitis, $\mathrm{n}=3$ both small (SB) and large bowel disease, $n=1$, only SB disease) while 2 had ulcerative colitis. 3 patients with CD had previously undergone surgery. The predominant indication was assessment of disease activity. In patients who had a successful test, mucosal visualisation enabled adequate assessment of disease activity in all (100\%), thus guiding effective therapy.

In suspected IBD patients, majority had diarrhoea (100\%) and abdominal pain (63.2\%). Pathology was detected in $63.2 \%$ of patients. SB Crohn's was diagnosed in 4 (21.1\%) patients while proctitis was noted in 1 (5.3\%). Other significant findings included non-steroidal anti-inflammatory medication related injury (5.3\%), colonic polyps (10.5\%), angioectasia (21.1\%) and diverticular disease (26.3\%).

Capsule transit times in the SB and colon were 71 (IOR 47-136) and 80 (IOR 48.5-216.5) min respectively; bowel cleanliness was 2 (IQR 2-3)).

Conclusion In patients with proven or suspected IBD, CCE is an effective investigation modality. Its strengths lie in its minimally invasive nature coupled with its ability to assess disease activity thus guiding treatment strategies in most cases.

Competing interests None.

Keywords colon capsule, Crohn's disease, inflammatory bowel disease, PillCam, ulcerative colitis. 